\title{
Search for Fermionic Higgs Boson Decays in $p p$ Collisions at the ATLAS and CMS Experiments
}

\author{
Romain Madar on behalf of the ATLAS and CMS Collaborations \\ Physikalisches Institut, Albert-Ludwigs-Universität, Hermann-Herder-Str. 3 \\ Freiburg 79104, Germany \\ romain.madar@cern.ch \\ Received Day Month Year \\ Revised Day Month Year
}

\begin{abstract}
The newly discovered boson was only observed in diboson final states. The measurement of the fermionic decay modes of this new particle is essential to establish its compatibility with the Standard Model Higgs boson. The two main final states accessible at the LHC are the decay into pairs of $b$ quarks and $\tau$ leptons, both of which are experimentally challenging. In some cases, the exploitation of specific Higgs boson production modes allows to significantly increase the experimental sensitivity. One can also extend the search by looking for di-muon final states. This article gives an overview of the Higgs boson searches in these three fermionic final states at the ATLAS and CMS experiments, focusing on the most recent developments and results.
\end{abstract}

\section{Introduction}

The Higgs boson $(H)$ is the relic particle of the electroweak symmetry breaking and is of fundamental interest. Since the discovery of the Higgs boson ${ }^{3,4}$ by the ATLAS and CMS experiments ${ }^{1,2}$, it is known that this new particle couples to bosons while there is only indirect evidence of couplings to fermions (via loops). The role of direct searches for fermionic Higgs boson decays is to directly measure these couplings. Also, the experimental uncertainty on the Higgs boson branching ratios is highly dominated by the most important decay mode, $H \rightarrow b b$. As a consequence, improving the precision on this branching fraction will reduce the uncertainty on all Higgs boson decay widths. Finally, loop induced production modes involve fermions but might also have contributions due to still unknown particles. Thus, a direct measurement of fermionic couplings would allow to disentangle New Physics (NP) from Standard Model (SM) contributions of these loop diagrams.

The overall analysis strategy is motivated in Section 2. Sections 3, 4 and 5 describe the search for the $b \bar{b}, \tau \tau$ and $\mu \mu$ final states respectively, while Section 6 focuses on searches dedicated to the $t t H$ production mode. 


\section{Overview of the search strategy}

Assuming a mass of $m_{H}=125 \mathrm{GeV}$, the total cross section of the $p p \rightarrow H+X$ process is of the order of $\sigma_{\text {tot }} \sim 20 \mathrm{pb}$, with four main contributing processes. The gluon fusion (GGF), via heavy quark loops, gives the highest contribution (88\% of $\sigma_{\text {tot }}$ ) but doesn't have any specific event topology allowing to reject the background. The vector boson fusion process (VBF) has a lower contribution $\left(6.6 \%\right.$ of $\left.\sigma_{\text {tot }}\right)$ but is a tree level process with two forward jets in the final state, offering a very specific signature. The production in association with a vector boson has a low contribution ( $5 \%$ of $\sigma_{\text {tot }}$ ) but has a clean final state due to lepton(s) from the vector boson decay. The production in association with a $t \bar{t}$ pair has an extremely low contribution $(0.4 \%$ of $\sigma_{\text {tot }}$ ) and a busy environment, but its measurement provides a unique access to the coupling between the top quark and the Higgs fields.

The Higgs boson decay is dominated by the $b \bar{b}$ final state $\left(\mathcal{B}_{b \bar{b}}=57 \%\right)$ which is very difficult to extract from the large non-resonant $p p \rightarrow b \bar{b}+X$ background $\left(\sigma_{b \bar{b}} \sim 10^{7} \mathrm{pb}\right)$. However, this decay mode can be identified using the signature of the leptonically decaying vector boson produced in association with $H$. The second important decay mode is the $\tau \tau$ final state $\left(\mathcal{B}_{\tau \tau}=6 \%\right)$ which, due to specific $\tau$ signatures, can be better separated from the multijet background . This final state also suffers from a high background contribution, especially for hadronic $\tau$ decay $\left(\tau_{\text {had }}\right)$ final states. However, the jet topology of the VBF production mode or highly boosted Higgs bosons recoiling against a jet allow to significantly increase the sensitivity of this channel. The Higgs boson can also decay to a $c \bar{c}$ pair with a comparable rate $\left(\mathcal{B}_{c \bar{c}}=6 \%\right)$, but this final state is extremely difficult to access in hadronic collisions. Finally, the di-muon final state benefits from a very good mass resolution, but suffers from an extremely low branching ratio $\left(\mathcal{B}_{\mu \mu}=0.02 \%\right)$ together with a large background from the $Z$ production $\left(\sigma_{\mathrm{Z}} \sim 10^{4} \mathrm{pb}\right)$.

Table 1. Overview of the final states and production modes analysed and the integrated luminosity used for both the ATLAS and CMS experiments.

\begin{tabular}{ccccc}
\hline & ATLAS & & CMS & \\
& $\mathcal{L}\left[\mathrm{fb}^{-1}\right](7 / 8 \mathrm{TeV})$ & Ref. & $\mathcal{L}\left[\mathrm{fb}^{-1}\right](7 / 8 \mathrm{TeV})$ & Ref. \\
\hline$H \rightarrow b b$ & & & & \\
\hline $\mathrm{VBF}$ & $-/-$ & $-/-$ & $-/ 19.0$ & Ref. 5 \\
$\mathrm{VH}$ & $4.7 / 20.3$ & Ref. 6 & $5.0 / 19.0$ & Ref. 7 \\
$\mathrm{ttH}$ & $4.7 /-$ & Ref. 6 & $-/ 19.0$ & Ref. 9 \\
\hline \multicolumn{1}{l}{$H \rightarrow \tau \tau$} & & & & \\
\hline $\mathrm{GGF}+\mathrm{VBF}$ & $4.9 / 13.0$ & Ref. 10 & $4.9 / 19.4$ & Ref. 11 \\
$\mathrm{VH}$ & $-/-$ & - & $-/ 19.0$ & Ref. 12 \\
$\mathrm{ttH}$ & $-/-$ & - & $-/ 19.0$ & Ref. 9 \\
\hline$H \rightarrow \mu \mu$ & & & & - \\
\hline Inclusive & $-/ 20.7$ & Ref. 13 & $-/-$ & - \\
\hline
\end{tabular}


The overall strategy is then to exploit all possible combinations of Higgs boson production and decay modes. A summary of the presented searches is given in Table 1. The following section gives more details about each search channel.

\section{Search for $H \rightarrow b b$}

The $b \bar{b}$ final state exploits all production modes except GGF, as explained in Section 2. The VBF production mode leads to a four jets final state. The large multijet background can be reasonably reduced exploiting the jet topology of the signal process. The associated production takes advantage of the vector boson decay signature and is clearly the most sensitive channel for this decay mode. Finally, the search for the $t t H(\rightarrow b \bar{b})$ process is described Section 6 .

Independently of the production mode, the key observable for $H \rightarrow b \bar{b}$ searches is the invariant mass of the $b \bar{b}$ system $\left(m_{b \bar{b}}\right)$, since the Higgs boson would appear as an excess of events close to $m_{H}$. The energy scale and resolution of $b$-quarks are then crucial for the experimental sensitivity. Both experiments achieved significant improvements by refining their treatment of the $b$-jet energy calibration: the kinematic dependence of the $b$-jet energy response is now taken into account and the energy correction due to $B$-hadron semi-leptonic decays is also added. These modifications, applied in a slightly different way in the two experiments, lead to a 10 to $15 \%$ improvement on the $m_{b \bar{b}}$ resolution, depending on the phase space region.

\subsection{Optimized search for the associated production}

For both experiments, the general strategy is to categorize the events according to the number of reconstructed leptons $n_{\ell}(\ell \equiv e, \mu)$ : the 0-lepton channel is enriched in $Z(\rightarrow \nu \nu) H(\rightarrow b \bar{b})$ events, the 1-lepton channel is enriched in $W(\rightarrow \ell \nu) H(\rightarrow$ $b \bar{b})$ events and the 2-lepton channel is enriched in $Z(\rightarrow \ell \ell) H(\rightarrow b \bar{b})$ events. The neutrinos, coming from $W$ and $Z$ decays, don't interact with the detector and will be indirectly measured via the missing transverse energy $E_{T}^{\text {miss }}$, i.e. as a momentum imbalance in the plane transverse to the beam axis.

The main Standard Model processes contributing to these final states are the $t \bar{t}$ pair production (leading to a $b \bar{b}$ pair and $b c$ pairs with a misidentified $c$-quark), the $W / Z+$ light quarks production (due to $b$-jet mis-identification), the $W / Z+b \bar{b}$ production leading to an identical final state, and the multi-jet production with and without real $b$-quarks (due to fake and/or non isolated lepton, in addition to misidentified $b$-jet). Several kinematic properties of these backgrounds offer a way to identify signal enhanced phase space regions. In addition to the $b \bar{b}$ system invariant mass, considering events with high transverse momentum of the vector boson $\left(p_{T}^{V}\right)$ allows to increase the signal-to-background $(S / B)$ ratio. Both experiments follow the same strategy to achieve a reliable and precise background modelling. First, several regions with different background and signal compositions are defined, using $n_{\ell}$, the number of reconstructed jets $\left(n_{\text {jet }}\right)$ and $b$-jets $\left(n_{b}\right)$ as well as $p_{T}^{V}$. Then, different 
parameters affecting the background prediction are defined, such as normalisation factors or continuous parameters modelling the effect of a given systematic uncertainty on the background. Finally, a global fit to the observed data is performed in order to determine the set of parameters which describes the data best, simultaneously in all regions. In this way, correlations of systematic variations across the regions are taken into account, the signal contamination in low $S / B$ regions is properly propagated and the various background compositions of the samples ensure that every single background is constrained.

The strategy to further reduce the background on top of existing categorisation, is to exploit differences in event topology between signal and backgrounds. On top of $b \bar{b}$ system properties, the angle between $E_{T}^{\text {miss }}$ and the $b \bar{b}$ system (expected to be around $\pi$ for $Z(\rightarrow \nu \nu) H(\rightarrow b \bar{b}))$, or the compatibility of the $\left(\ell, E_{T}^{\text {miss }}\right)$ system with a $W$ decay (as expected for $W(\rightarrow \ell \nu) H(\rightarrow b \bar{b}))$ are also used. The ATLAS search is performed by analysing the $m_{b b}$ distribution after cut-based selections on these variables while the CMS search combines them in a Boosted Descision Tree (BDT) which is used as discriminant observable.

An important proof of principle of any experimental search is to be able to measure a known process having the same signature as the signal. The $V H(\rightarrow b \bar{b})$ search can be validated by measuring the SM production of $V Z$ pairs. Both the ATLAS and CMS experiments are able to measure $V Z$ production at the expected rate.
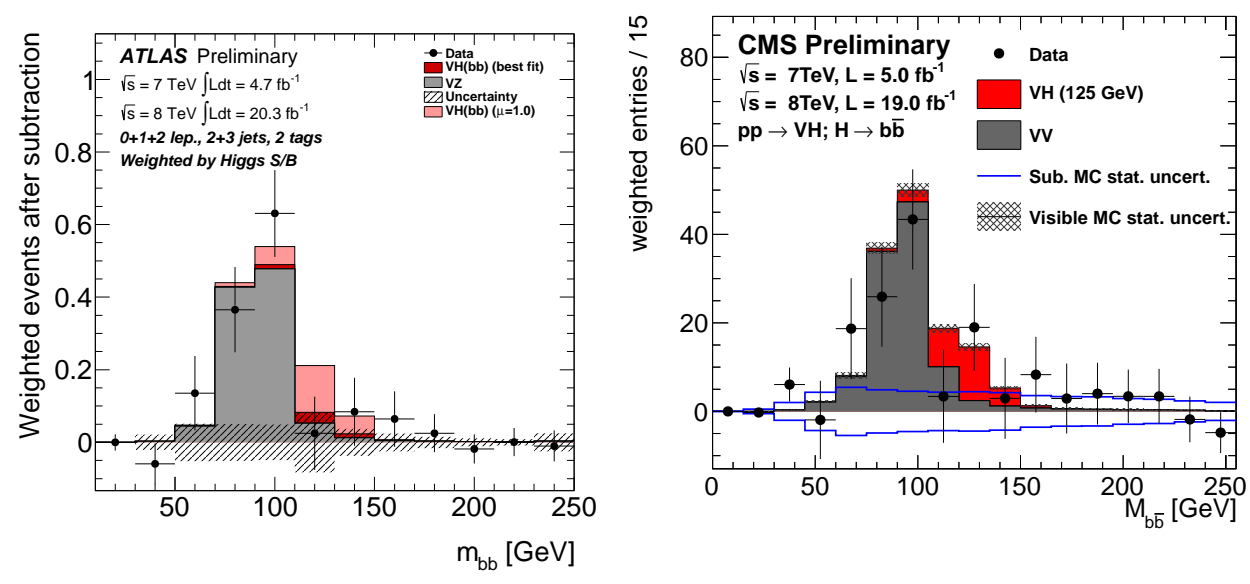

Fig. 1. Weighted $m_{b b}$ distribution after subtraction of the non-resonant backgrounds for the ATLAS (left) and CMS (right) experiments.

Figure 1 shows the final $m_{b b}$ invariant mass distribution after subtraction of the non-resonant backgrounds. The events are weighted according the expected $S / B$ in each bin of the $m_{b b}$ distribution. To interpret the result, the signal strength $\mu$ is 
defined as the ratio between the observed (fitted) cross section and the expectation from the SM. The ATLAS experiment quotes an observed (expected) exclusion limit at $95 \%$ confidence level on $\mu$ of $1.3(1.4)$ for $m_{H}=125 \mathrm{GeV}$ and an extracted signal strength of

$$
\mu=0.2 \pm 0.6 .
$$

The CMS experiment quotes an observed (expected) exclusion limit at $95 \%$ confidence level on $\mu$ of 1.7 (0.94) for $m_{H}=125 \mathrm{GeV}$, and an extracted signal strength of

$$
\mu=1.0 \pm 0.5 .
$$

The ATLAS result is compatible with both the signal and background only hypothesis, while the CMS experiment has an excess in the observed data with respect to the expected background of about two standard deviations.

\subsection{Optimized search for the vector boson fusion production}

The search for $q q H(\rightarrow b \bar{b})$ is particularly challenging because of the important multijet background. The CMS experiment has designed a dedicated four-jet final state trigger for this search, taking advantage of the two VBF forward jets, namely with a high rapidity gap and a high invariant mass. The other key signature is the presence of two $b$-jets inside this rapidity gap. Finally, to further reduce the multijet background where the two forward jets are due to gluon radiation, a $q / g$ jet discriminant is built based on the expected differences between quark and gluon jet shapes. In order to efficiently exploit the full information, the $b$-jet identification variable, the VBF tagging jet variables and the $q / g$-jet discriminant are combined in a neural network (NN). Four analysis categories are defined based on the NN output. For each category, an unbinned fit of the $m_{b b}$ distribution is performed to test the presence of a signal. The final result, given by the combination of these categories, reaches an observed (expected) limit on $\mu$ at the $95 \%$ confidence level of 3.6 (3.0) for $m_{H}=125 \mathrm{GeV}$. Figure 2 shows the distribution of the NN output and the expected and observed limit versus $m_{H}$.

\section{Search for $H \rightarrow \tau \tau$}

The $\tau \tau$ final state exploits all production modes. This final state is sensitive to the GGF process mainly for high transverse momentum of the $\tau \tau$ system, called boosted topology. However, also this search is most sensitive to the VBF production, using the tagging jets to significantly reduce the $Z \rightarrow \tau \tau$ background. The associated production provides an appreciable sensitivity in spite of its lower rate. Finally, the search for the $t t H(\rightarrow \tau \tau)$ process is described Section 6.

On the other hand, analysing di- $\tau$ final states is experimentally challenging for several reasons. First, each $\tau$-lepton decays into stable particles before reaching 

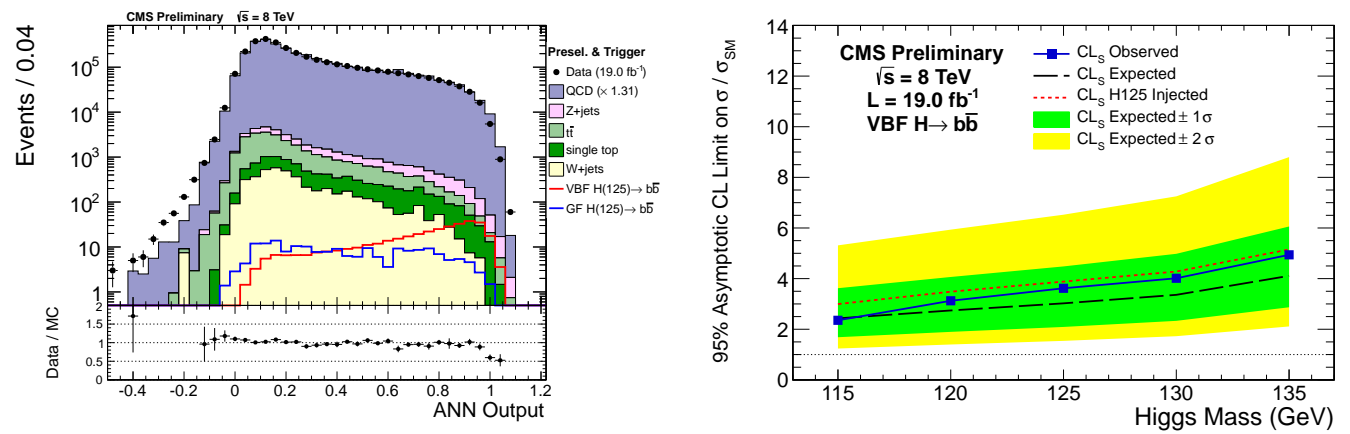

Fig. 2. NN distribution used to define the five categories: $N N<0.52,0.52<N N<0.76$, $0.76<N N<0.90,0.90<N N<0.96$ and $N N>0.96$ (left). Expected and observed exclusions limit on $\mu$ at the $95 \%$ confidence level (right). The expected limits are shown for the background only hypothesis as well as for the signal plus background hypothesis (signal of $m_{H}=125 \mathrm{GeV}$ injected).

the detector. This leads to three different final states with different background compositions: $\tau_{\text {had }} \tau_{\text {had }}(41.9 \%), \tau_{\ell} \tau_{\ell}(12.4 \%)$ and $\tau_{\ell} \tau_{\text {had }}(45.8 \%)$, where $\tau_{\ell}$ denotes the lepton $\ell$ of the $\tau \rightarrow \ell \nu_{\tau} \nu_{\ell}$ decay and $\tau_{\text {had }}$ generically denotes the hadronic decay products of the $\tau \rightarrow$ hadrons $\nu_{\tau}$ decay. Each of these final states needs a dedicated analysis in order to maximize the overall sensitivity. Secondly, the measurement of the di- $\tau$ invariant mass $\left(m_{\tau \tau}\right)$ is particularly challenging due to the escaping neutrinos. In both the ATLAS and CMS experiments, the kinematic of the neutrinos is assessed given the observed missing transverse energy and the matrix element of the $\tau$-lepton decay. This kinematic fit, applied in a slightly different way in the two experiments, leads to an improvement of the order of $25 \%$ in the sensitivity due to a better $m_{\tau \tau}$ resolution. Finally, it is important to keep the energy response of hadronically decaying $\tau$ lepton under control, which is achieved within a precision of the order of $\pm 5 \%^{14}$.

\subsection{Optimized search for gluon fusion and vector boson fusion}

The common strategy is to search for an excess of events localized around $m_{\tau \tau}=$ $125 \mathrm{GeV}$ on top of the $Z \rightarrow \tau \tau$ background. The estimation of this most important background is based on $Z \rightarrow \mu \mu$ data events for both experiments. After selection of a pure data sample of $Z \rightarrow \mu \mu$, muon energy deposits are removed and are replaced by those of simulated $\tau$ with the kinematics as the initial muons. In this way, the so-called embedding technique, the jet kinematics, the detector noise and all the soft interactions are directly taken from data. Furthermore, the hadronic $\tau$ final states suffer from background due to jets wrongly identified as $\tau_{\text {had }}$, such as $W(\rightarrow \ell \nu)+$ jets where one jet is misidentified as a $\tau_{\text {had }}$. In order to better control the fake backgrounds, data-driven estimates are used. In the ATLAS $\tau_{\text {had }} \tau_{\text {had }}$ analysis, the multijet normalisation is obtained by performing a two-dimensional template fit 
to the track multiplicity distribution of the two $\tau_{\text {had }}$ candidates, where the multijet template is modelled from data using same sign electric charge candidates. In the CMS analysis, the multijet background is estimated using a non isolated $\tau_{\text {had }}$ control sample in data.

To optimize the sensitivity, the events are categorized following the two main topologies in a similar way for all three sub-channels:high momentum of the $(\tau, \tau)$ pair (sensitive to GGF) and high invariant mass between two forward jets (sensitive to $\mathrm{VBF}$ ). This last topology heavily relies on jet kinematic, properties which are modelled using data ensuring the background estimation robustness in this most sensitive phase space region. To further suppress the backgrounds, different properties can be exploited depending on the process to reject. For example, the missing transverse energy points in the direction between the two $\tau$-lepton candidates for $Z / H \rightarrow \tau \tau$, but not for non-resonant backgrounds like $W+$ jets or $t \bar{t}$ production. The ATLAS and CMS experiments exploit this property using the transverse mass of the lepton candidate and the missing energy $\left(m_{T}\left(\ell, E_{T}^{\mathrm{miss}}\right)\right)$ and the $E_{T}^{\mathrm{miss}}$ projection on the direction of the $\tau \tau$-axis, respectively.
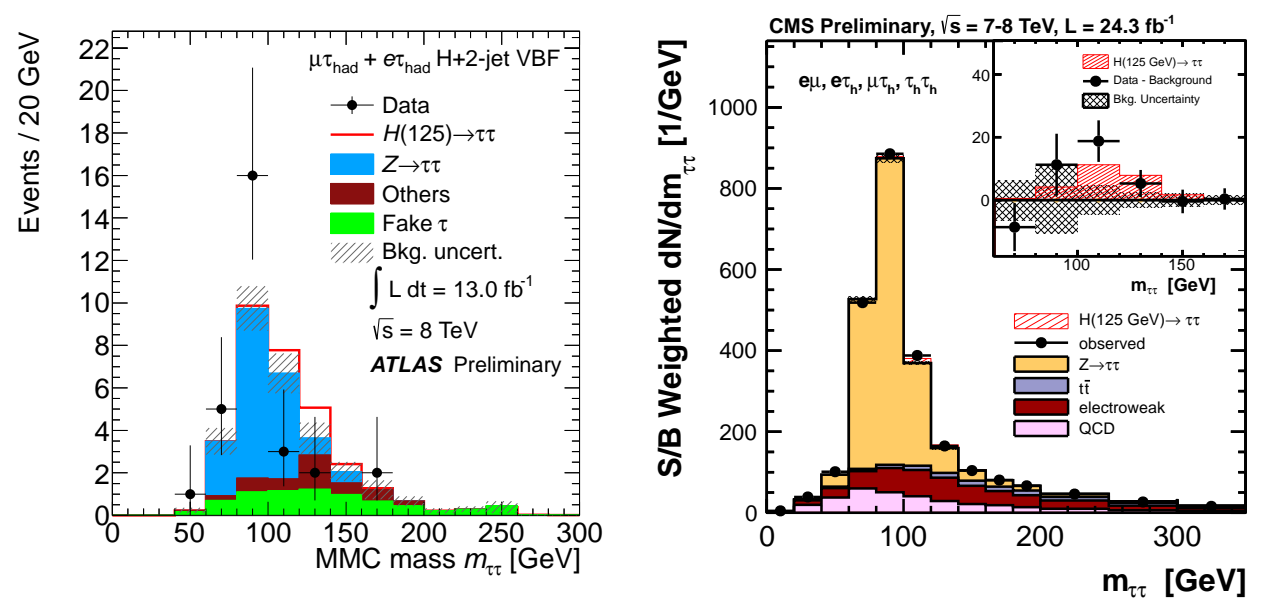

Fig. 3. Invariant mass distribution of the $\tau \tau$ system for the VBF category in the $\tau_{\ell} \tau_{\text {had }}$ final state at the ATLAS experiment (left). $S / B$ weighted invariant mass distribution of the $\tau \tau$ system for four channels at the CMS experiment (right). The upper box represents the background-subtracted weighted data overlaid with a $125 \mathrm{GeV}$ signal.

By combining the three $\tau \tau$ final states, the ATLAS analysis reaches an observed (expected) limit on $\mu$ at $95 \%$ confidence level of $1.9(1.2)$ for $m_{H}=125 \mathrm{GeV}$. The CMS analysis achieves an observed (expected) limit of 0.77 (1.8) at the same mass. The ATLAS and CMS experiments measures a signal strength of $\mu=0.7 \pm 0.7$ and $\mu=1.1 \pm 0.4$, respectively. For CMS, this corresponds to a 2.8 standard deviation excess of observed events compatible with a $125 \mathrm{GeV}$ Higgs boson. The ATLAS 
result is compatible with both the signal and the background-only hypothesis. The ATLAS collaboration is currently working on an update of this search to inlude the full available dataset. Figure 3 shows the invariant mass distributions of the di- $\tau$ system for both analyses.

\subsection{Optimized search for the associated production}

Even if vector boson (semi)-leptonic decays only is considered, the combinatorics due to the three $\tau \tau$ pair decays lead to several possible final states. The analysed channels are then chosen taking into account hadronic $\tau$ identification efficiency, branching ratios and background rates. The CMS experiment analyses the following channels: $\ell \ell \tau_{\text {had }}$ enriched in $W(\rightarrow \ell \nu) H\left(\rightarrow \tau_{\ell} \tau_{\text {had }}\right)$, $\ell \ell \ell \ell$ enriched in $Z(\rightarrow \ell \ell) H\left(\rightarrow \tau_{\ell} \tau_{\ell}\right)$ and $\ell \tau_{\text {had }} \tau_{\text {had }}$ mostly enriched in $W(\rightarrow \ell \nu) H\left(\rightarrow \tau_{\text {had }} \tau_{\text {had }}\right)$. The charge and/or the flavour correlation between the different particles represents a valuable information to reduce the background. The most important SM backgrounds are the $W Z$ and $Z Z$ production for $\ell \ell \tau_{\text {had }}$ and $\ell \ell \ell \ell$ channels, respectively, while the fake background is dominant in the $\ell \tau_{\text {had }} \tau_{\text {had }}$ final state. The signal extraction is based on the invariant mass spectrum of all the visible decay products of the reconstructed Higgs boson candidate. By combining the different channels, this search reaches an interesting sensitivity:the observed (expected) limit on $\mu$ at $95 \%$ confidence level is 3.1 (3.9) for $m_{H}=125 \mathrm{GeV}$, as illustrated Figure 4.
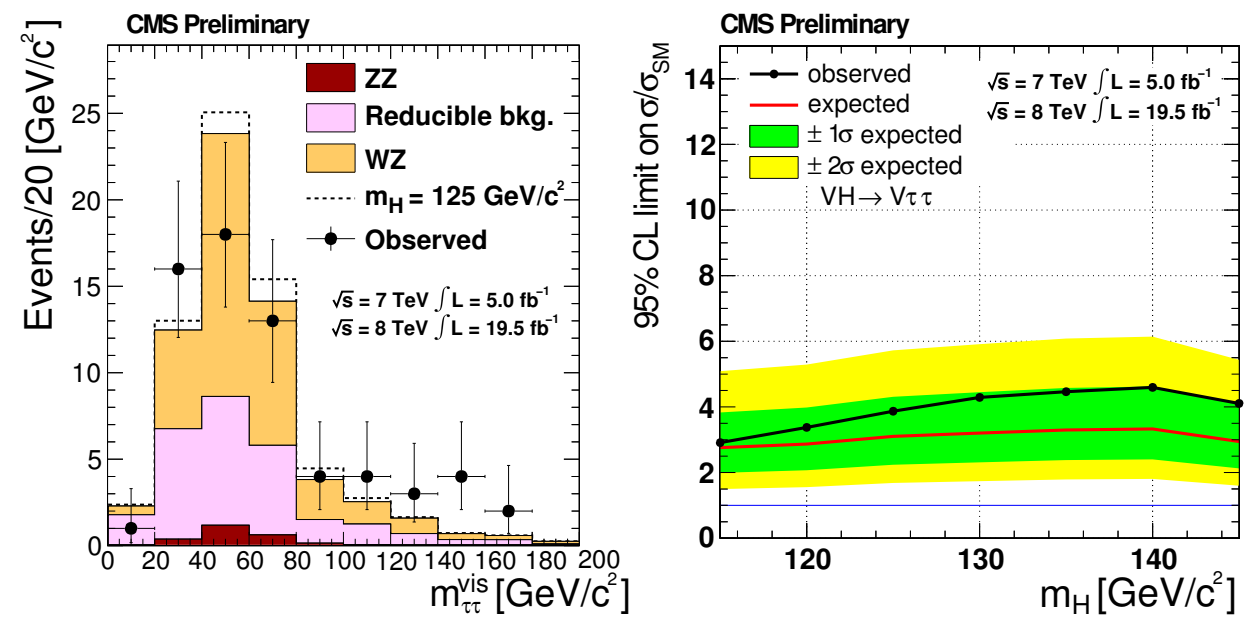

Fig. 4. Invariant mass of the $\tau_{\ell} \tau_{\text {had }}$ system, for the $\ell \ell \tau_{\text {had }}$ final state as measured in the CMS experiment (left). Expected and observed exclusion limits on the signal strength for different $m_{H}$ (right). 


\section{Search for $H \rightarrow \mu \mu$}

The search for $H \rightarrow \mu \mu$ is extremely challenging because of the low branching ratio of this decay mode and the large cross section of the $Z \rightarrow \mu \mu$ background. However, the good experimental resolution of the di-muon mass $\left(m_{\mu \mu}\right)$ leads to a promising sensitivity $\left(S / \sqrt{B} \sim 0.25\right.$ for $20 \mathrm{fb}^{-1}$ ). In addition, this channel offers a unique way to directly probe the coupling between the Higgs boson and second generation fermions. Finally, the $H \rightarrow \mu \mu$ branching ratio is sensitive to several SM extensions ${ }^{13}$, such that this channel becomes valuable also for new physics searches.

The analysis strategy is to search for an excess of events at $m_{\mu \mu} \sim m_{H} \mathrm{GeV}$. The events are split into two categories based on the $m_{\mu \mu}$ resolution. The background and signal modelling relies on analytical $m_{\mu \mu}$ shapes, determined using simulated distributions. The signal hypothesis is tested via an unbinned fit of $m_{\mu \mu}$. The observed (expected) sensitivity in terms of signal strength is 9.8 (8.2) for $m_{H}=125 \mathrm{GeV}$, as illustrated Figure 5.
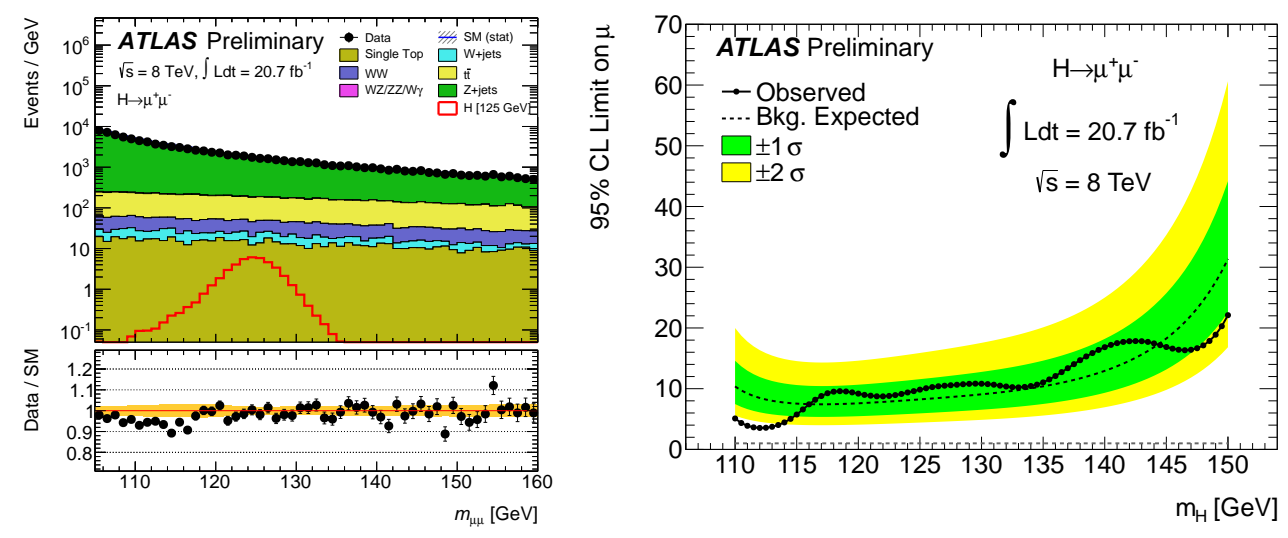

Fig. 5. Invariant mass of the di-muon system as measured in the ATLAS experiment (left). Expected and observed exclusion limits on the signal strength for different $m_{H}$ (right).

\section{Dedicated searches for $t \bar{t} H$ production}

Even though the $t t H$ production represents only $0.4 \%$ of the total production cross section, the search for this process has several motivations. First, it provides direct access to the couplings between the Higgs boson and the top quark. This direct measurement, together with the $g g \rightarrow H$ cross-section measurement, could allow to constrain new physics involved in the loops of this process. Finally, it allows to test Higgs field properties at a higher mass scale where new physics is more likely to appear. However, this channel is experimentally challenging mainly because of a complex final state containing electrons, muons or $\tau$-leptons, four $b$-jets and missing 
transverse energy. Some of the SM backgrounds, like $t \bar{t}+b$-jets and $t \bar{t}+W / Z$, are not well known and the associated systematic uncertainties can significantly degrade the search sensitivity.

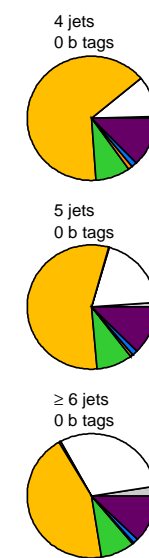

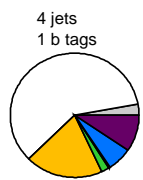

5 jets
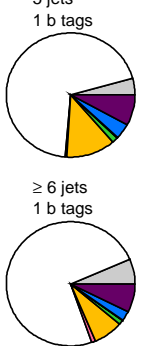

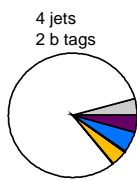

5 jets
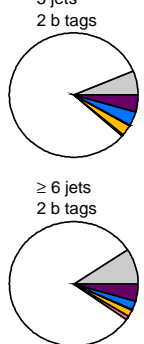

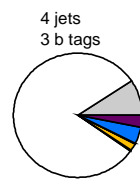

5 jets

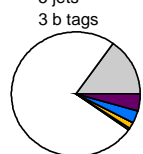

$\geq 6$ jets

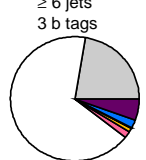

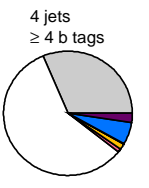

5 jets

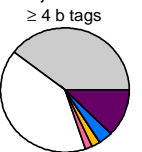

$\geq 6$ jets

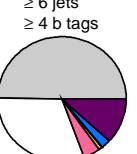

\section{ATLAS}

Preliminary

(Simulation)

$\mathrm{m}_{\mathrm{H}}=125 \mathrm{GeV}$
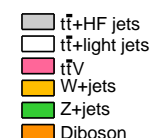

Diboson

Single to
Multijet

Fig. 6. Expected background composition in the ATLAS $t t H(\rightarrow b \bar{b})$ analysis, for each defined category based on the number of reconstructed jets and $b$-jets.

In order to cope with these background uncertainties, both the ATLAS and CMS collaborations follow the same general strategy defining different phase space regions enriched in different backgrounds and performing a global fit to data in order to constrain each single background. Figure 6 illustrates how these samples are defined and what the expected background compositions for the ATLAS $t t H(\rightarrow b \bar{b})$ analysis are.

\subsection{ATLAS searches}

The ATLAS search focuses on $H \rightarrow b \bar{b}$ decay and the semi-leptonic decay of the $t \bar{t}$ pair, analysing the $\ell \nu+4 b$-jets +2 jets final state. A search for $t t H(\rightarrow \gamma \gamma)^{15}$ is also performed but is not presented in this document. One of the specific challenge of this final state is the event kinematic reconstruction. Indeed, among the four reconstructed $b$-jets, it is a priori impossible to identify the pair coming from the Higgs boson decay. The ATLAS experiment developed a method based on an eventby-event kinematic fit in order to determine the $b$-jet pair most likely coming from the Higgs boson decay. As shown in Figure 6, the background modelling is done by defining categories based on the number of reconstructed jets and $b$-jets. The global fit allows to significantly reduce the $t \bar{t}+b$-jets and the $t \bar{t}+W / Z$ background uncertainties. For each of these categories, the invariant mass of the $b$-quark pair, identified as coming from $H \rightarrow b \bar{b}$, is used to test the presence of a signal. After combining all categories, this analysis achieves an observed (expected) limit on $\mu$ 
of $13.1(10.5)$ for $m_{H}=125 \mathrm{GeV}$ as illustrated in Figure 7.
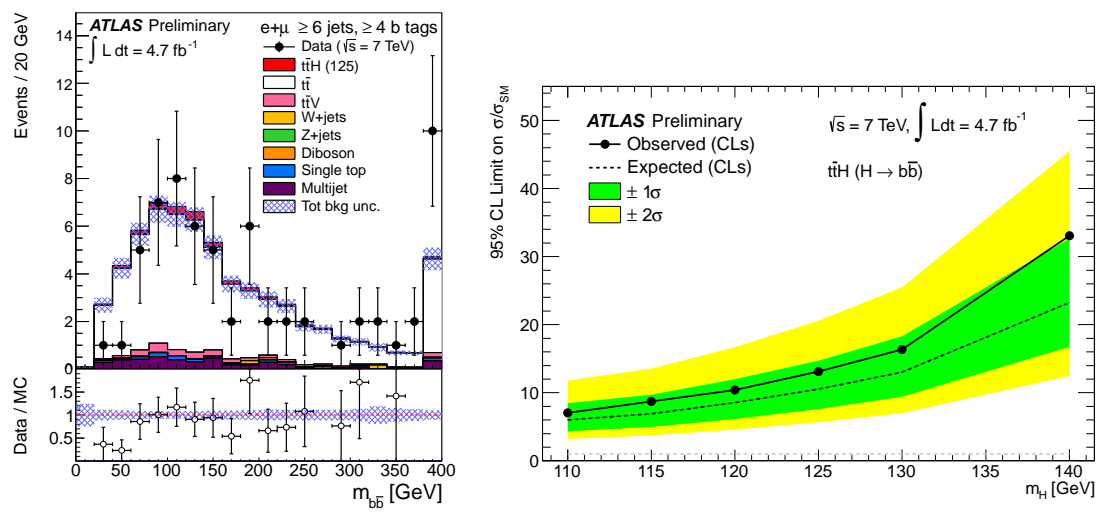

Fig. 7. Invariant mass of the $b$-quark pair identified as coming from $H \rightarrow b \bar{b}$ as measured in the ATLAS experiment(left). Expected and observed exclusion limit on the signal strength for different $m_{H}$ (right).

\section{2. $C M S$ searches}
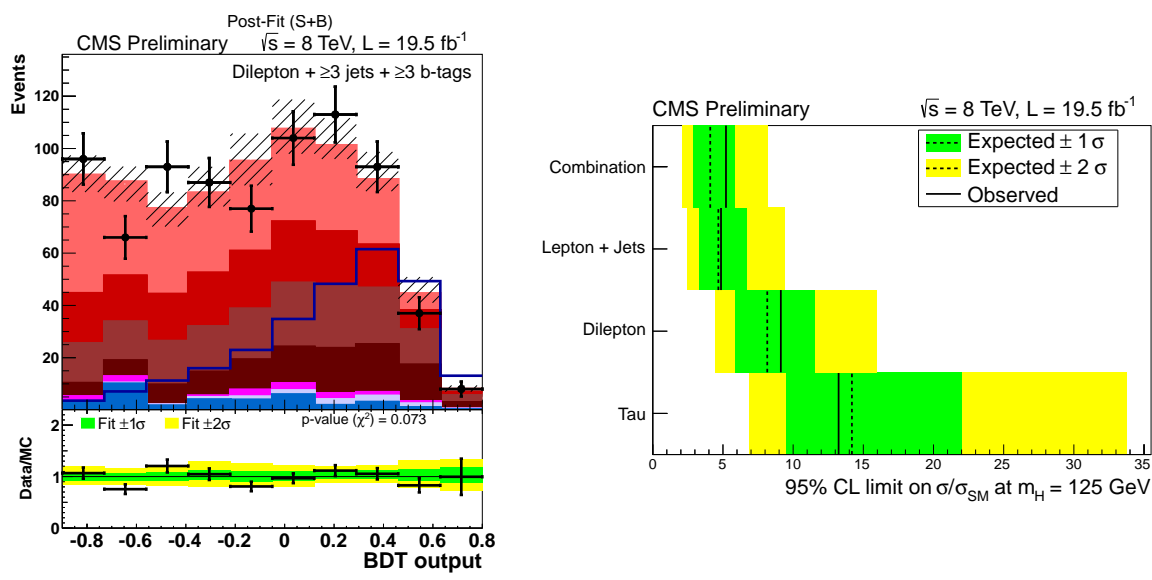

Fig. 8. BDT output distribution as measured in the CMS experiment (left). Expected and observed exclusion limit on the signal strength for different $m_{H}$ (right).

The CMS searches focus on $H \rightarrow b \bar{b}$ and $H \rightarrow \tau \tau$ decays together with semileptonic and di-leptonic decays of the $t \bar{t}$ pair. The $H \rightarrow \gamma \gamma$ decay is also analysed and a $H \rightarrow b b / \tau \tau / \gamma \gamma$ combination is available as an inclusive search for $t t H$, but is not presented in this document. The two $t \bar{t}$ decays are treated as different categories 
for the $H \rightarrow b \bar{b}$ search while they are treated inclusively for the $H \rightarrow \tau \tau$ search. The signal is extracted using various discriminant variables combined in a BDT, like $b$ quark and $\tau$-lepton identification variables as well as $b$-jet pairing related variables. By combining the three channels, this analysis yields an observed (expected) limit of 5.2 (4.1) for $m_{H}=125 \mathrm{GeV}$. The improvement provided by the $\tau \tau$ channel is illustrated Figure 8.

\section{Conclusions}

Higgs boson fermionic decay modes are experimentally challenging to extract in the presence of large backgrounds. However, they are crucial to fully understand the electroweak symmetry breaking sector and how the Higgs boson couples to elementary fermions. The results of these searches constitute an essential input to identify the nature of the newly discovered Higgs boson. A wide LHC program to address this fundamental question was designed and first exciting results are obtained by the ATLAS and CMS experiments in the $H \rightarrow b \bar{b}$ and $H \rightarrow \tau \tau$ decay modes. A 3.4 standard deviation excess in the combined $H \rightarrow \tau \tau$ and $H \rightarrow b b$ searches from the CMS collaboration is observed. The result obtained by the ATLAS collaboration is compatible with the signal hypothesis as well as the backgroundonly hypothesis. An update of the ATLAS search inluding the full available dataset is under preparation. In addition, some valuable secondary channels reach very promising sensitivities and are expected to contribute to our understanding of the Higgs sector in the upcoming years.

\section{References}

1. ATLAS Collaboration, JINST 3 S08003 (2008).

2. CMS Collaboration, JINST 3 S08004 (2008).

3. ATLAS Collaboration, Phys. Lett. B 716 1-29 (2012)

4. CMS Collaboration, Phys. Lett. B 71630 (2012)

5. CMS Collaboration, CMS PAS HIG-13-011

6. ATLAS Collaboration, ATLAS-CONF-2013-79

7. CMS Collaboration, CMS PAS HIG-13-012

8. ATLAS Collaboration, ATLAS-CONF-2012-135

9. CMS Collaboration, CMS PAS HIG-13-019

10. ATLAS Collaboration, ATLAS-CONF-2012-60

11. CMS Collaboration, CMS PAS HIG-13-004

12. CMS Collaboration, CMS PAS HIG-12-053

13. ATLAS Collaboration, ATLAS-CONF-2013-10

14. ATLAS Collaboration, ATLAS-CONF-2013-044

15. ATLAS Collaboration, ATLAS-CONF-2013-080 